\title{
Tuberculoma cerebral como única manifestación de tuberculosis en embarazada. Presentación de un caso
}

\author{
Cerebral tuberculoma as the only manifestation of tuberculosis in \\ pregnant women. Presentation of a case
}

\begin{abstract}
Ariel Varela Hernández'1, Patricio Herrera Astudillo', Félix Orellana Cortez', Luis Lamus Aponte', Claudio Martínez Terreu', Rodolfo Muñoz Gajardo', Reinaldo Torres Aravena', Jorge Becker Valdivieso', Renato Esteban Ocampo Avello ${ }^{3}$, Nélida Susel Isern Domínguez ${ }^{4}$, Julio Cesar Pérez Suárez, María Berenice García Gimenez
\end{abstract}

\footnotetext{
${ }^{1}$ Servicio de Neurocirugía. Hospital Regional de Talca.

${ }^{2}$ Servicio de Obstetricia y Ginecología. Hospital Regional de Talca.

${ }^{3}$ Servicio de Infectología. Hospital Regional de Talca.

${ }^{4}$ Servicio de Anatomía Patológica. Hospital Regional de Talca.

${ }^{5}$ Servicio de Imagenología. Hospital Regional de Talca.
}

\section{Resumen}

Introducción: Los tuberculomas cerebrales como manifestación exclusiva de tuberculosis, han sido raramente reportados durante el embarazo. Caso clínico: Se presenta el caso de una paciente con embarazo del segundo trimestre, sintomática de cefalea progresiva y diplopía. Los estudios neuroimagenológicos mostraron lesión tumoral frontal derecha, bien localizada, con extensa área de edema adyacente y pico lipídico en la espectroscopia. No se detectaron alteraciones en el resto de los aparatos y sistemas. Se realizó craneotomía y resección en bloque de la lesión a las 20 semanas de embarazo, sin complicaciones para la enferma ni para el feto, la biopsia comprobó el diagnóstico de tuberculoma. Recibió tratamiento antituberculoso por 6 meses. Presentó parto normal y actualmente está asintomática. Su hijo, de más de un año de edad en la actualidad, se mantiene sin alteraciones. Conclusiones: Los tuberculomas cerebrales como manifestación exclusiva de tuberculosis en el embarazo son lesiones raras pero probables. La sospecha clínica, la confirmación diagnóstica y la cirugía en los casos indicados, llevados a cabo con agilidad y planificados por un equipo multidisciplinario, son relevantes para la obtención de buenos resultados terapéuticos, tanto para la madre como para el feto.

Palabras clave: Tuberculoma intracraneal, embarazo, resonancia magnética del cerebro, craneotomía.

\section{Abstract}

Introduction: Cerebral tuberculomas, as an exclusive manifestation of tuberculosis, have been rarely reported during pregnancy. Clinical case: The case of a patient with a second trimester pregnancy with progressive headache and diplopia is presented. Neuroimaging studies showed right frontal tumor lesion, well located, with extensive area of adjacent edema and lipid peak in spectroscopy. No other alterations on physical exam were detected. Craniotomy and block resection of the lesion were performed at 20 weeks of pregnancy, without complications for the patient or the fetus, the biopsy verified the diagnosis of tuberculoma. He received tuberculosis treatment for 6 months. She had normal birth and is currently asymptomatic. His son, over one year old today, remains unchanged. Conclusions: Cerebral tuberculomas as an exclusive manifestation of tuberculosis in pregnancy are rare but probable. Clinical suspicion, diagnostic confirmation and surgery in the indicated cases, carried out with agility and planned by a multidisciplinary team, are relevant for obtaining good therapeutic results for both, the mother and the fetus.

Key words: Intracranial tuberculoma, pregnancy, MRI of the brain, craniotomy.

\section{Correspondencia a:}

Dr. Ariel Varela Hernández

varelahernandezariel@gmail.com 


\section{Introducción}

La tuberculosis (TB) se mantiene en la actualidad como una enfermedad reemergente a nivel mundial; hecho relacionado con el aumento de las migraciones a partir de zonas endémicas, la alta prevalencia de estados de inmunosupresión, como por ejemplo los relacionados con la infección por el virus de inmunodeficiencia humana y el desarrollo de resistencias a los fármacos antituberculosos ${ }^{14}$.

La tuberculosis extrapulmonar representa el $20-25 \%$ de todos los casos de tuberculosis y los tuberculomas solo el $1 \%$ de los mismos; esta es la forma clínica más rara de TB en el sistema nervioso. Dichas lesiones se presentan habitualmente secundarios a TB pulmonar o diseminada verificable, es rara su presentación como única manifestación de la enfermedad y en consecuencia su diagnóstico en estas circunstancias es pocas veces sospechado antes de la confirmación histopatológica ${ }^{11}$.

El reporte de tuberculomas durante el embarazo es parco. Aunque muchos autores no encuentran en esta condición un factor de riesgo para padecer la enfermedad, las variaciones inmunológicas que ocurren en este estado podrían contribuir con la generación de tuberculomas después de un segundo contacto de la paciente grávida con el Mycobacterium tuberculosis. Por otro lado, los propios síntomas relacionados con el embarazo pueden contribuir con la tardanza en el diagnóstico.

En el presente artículo presentamos el caso de una paciente con embarazo del segundo trimestre, la cual fue diagnosticada y operada de un tuberculoma cerebral, con evolución favorable tanto para la madre como para el feto.

\section{Caso clínico}

Paciente de 26 años, femenina, técnico de enfermería de
CESFAM, multípara, cursando embarazo de 19+5 semanas determinado por biometría. Antecedentes de contacto con tuberculoso bacilífero (en contexto laboral) en julio de 2017 , tras lo cual fue estudiada con baciloscopías, las cuales resultaron negativas.

Acudió derivada de otro centro hospitalario al Servicio de Urgencias del Hospital Regional de Talca (HRT) por presentar cefalea bifrontal progresiva, de tres meses de evolución, carácter gravativo, predominio nocturno y matutino, en ocasiones acompañada de vómitos. Comenzó con diplopía tres días previos al ingreso. No refería otros síntomas, incluyendo la esfera obstétrica.

Al examen físico neurológico se observó a la paciente con escala de Glasgow de 15 puntos, con oftalmoparesia del VI nervio craneano izquierdo, sin otros hallazgos. El examen físico obstétrico, así como del resto de los aparatos y sistemas fue normal.

Se interpretaron en ese momento imágenes de Tomografía Computarizada (TC) de cráneo sin contraste, tomadas en el hospital de origen, que mostraban zona hipodensa frontal derecha con efecto de masa, sugerente de lesión tumoral.

Aportaba también ecografía mamaria negativa (BIRADS 1). Ecografía abdominal: Leve esteatosis hepática. Útero grávido. Pruebas de bienestar fetal sin alteraciones. Determinaciones hematológicas y hemoquímicas generales dentro de parámetros normales. Anticuerpos anti-VIH negativos.

Se hospitalizó bajo medidas terapéuticas generales, analgesia y esteroidoterapia intravenosa. Durante el ingreso se tomaron imágenes de Resonancia Magnética (RM) del encéfalo, técnica estándar, sin contraste, complementada con espectroscopía, que evidenció lesión tumoral bien localizada, en región subcortical frontal derecha, rodeada de extensa zona de edema cerebral y luxación de línea media superior a los $4 \mathrm{~mm}$. En la espectroscopia llamó la atención la existencia de un pico lipídico (Figuras 1 y 2).

Se discutió el caso en un equipo multidisciplinario inte-

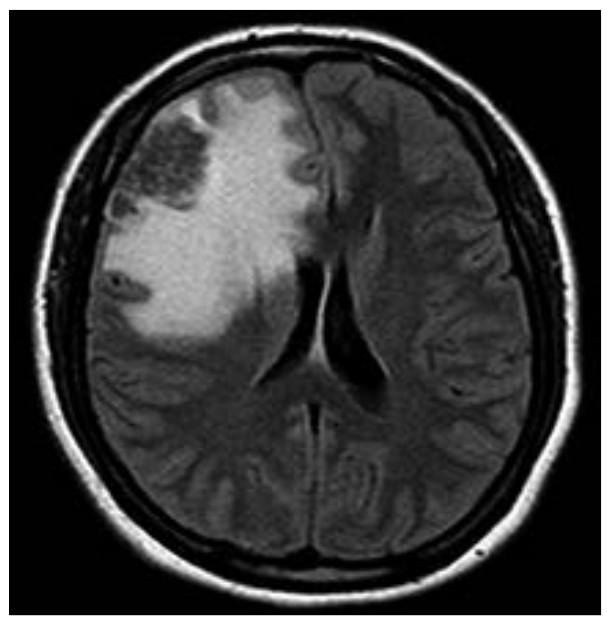

Figura 1. RM cerebral con técnica de FLAIR que muestra lesión tumoral, bien localizada, frontal derecha, con extensa área de edema cerebral adyacente y luxación de línea media.

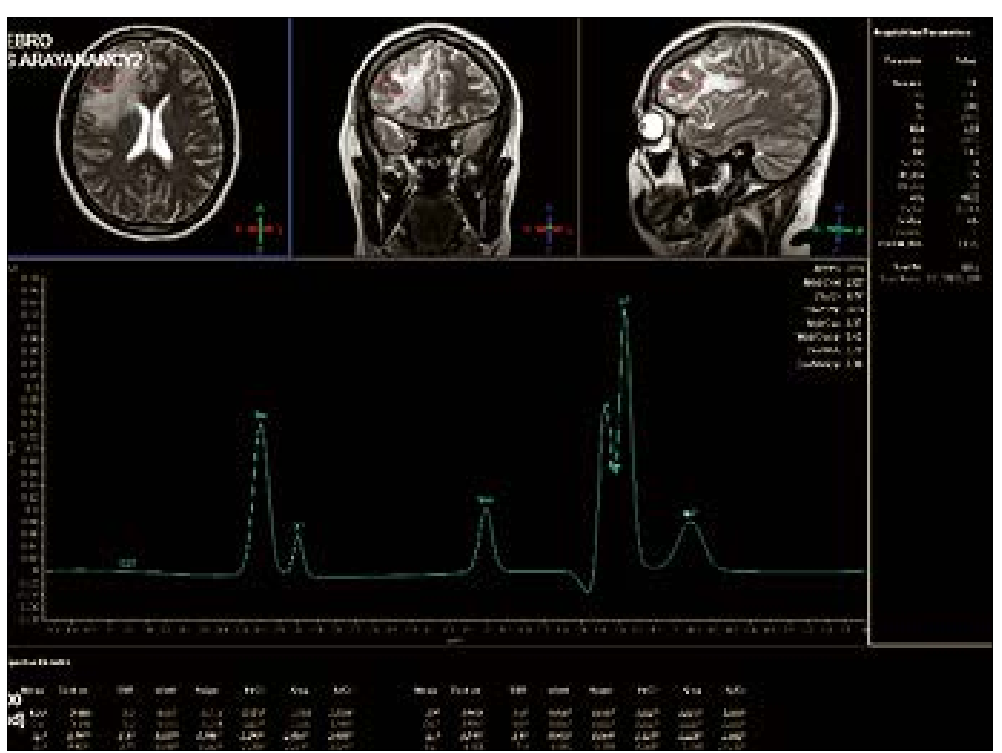

Figura 2. RM cerebral con técnica de espectroscopía que indica pico elevado de lípidos en la lesión tumoral frontal derecha descrita en figura 1. 
grado por el grupo de neurocirugía, obstetras, radiólogos, patólogos e infectólogos del HRT. Cursando las 20 semanas de embarazo se realizó craneotomía osteoplástica frontal derecha y la resección en bloque, apoyada con ultrasonografía cerebral transoperatoria, de la lesión subcortical superficial frontal derecha, encapsulada, poco vascularizada, de aspecto granulomatoso. No ocurrieron complicaciones transoperatorias ni postoperatorias.

Al cabo de dos semanas de la cirugía la paciente presentó nuevamente cefalea intensa y fiebre. No existía ninguna alteración evidente en la esfera neurológica ni obstétrica, pruebas de bienestar fetal normales. Se realizó RM cerebral de control: signos de edema en el área quirúrgica, sin evidencia de lesión tumoral residual ni recurrente. Se practicó punción lumbar (PL) para estudio del líquido cefalorraquídeo (LCR) que mostró pleocitosis a predominio linfocítico (leucocitos $361 / \mathrm{mm}^{3}, 82 \%$ mononucleares), asociada a hipoglucorraquia (26 mg/dl) y discreta hiperproteinorraquia (109 mg/dl), tinta china negativa. Adenosin deaminasa (ADA) dentro de rangos normales $(5,3 \mathrm{U} / \mathrm{I})$.

Paralelamente se obtuvo resultado de la biopsia de la lesión la cual mostro la presencia de un proceso inflamatorio crónico y necrotizante tipo tuberculoides con células gigantes tipo Langhans. La reacción de polimerasa en cadena (PCR) para Mycobacterium tuberculosis resulto positiva (Figura 3).

A partir de ese momento inicio tratamiento antituberculoso con esquema primario (isoniazida, rifampicina, pirazinamida y etambutol en dosis fijas combinadas), de acuerdo a Norma Técnica 2014 del Ministerio de Salud, el cual mantuvo por 6 meses. Además, durante las dos primeras semanas del tratamiento se asoció a dexametasona por vía oral (en dosis equivalente a $0,7 \mathrm{mg} / \mathrm{kg} /$ día). También se mantuvo con controles obstétricos frecuentes, según lo establece el programa de atención de estas enfermas.

Se produjo el parto de forma normal, a las 37 semanas, sin complicaciones. El recién nacido no mostro signos de TB congénita, peso al nacer de $3.450 \mathrm{Kg}$. En el momento actual la paciente culminó la terapia antimicrobiana, se mantiene libre de síntomas y su hijo, con más de 1 año de nacido, no ha presentado alteraciones.

\section{Discusión}

En relación a la TB pulmonar, la extrapulmonar es mucho menos frecuente, dentro de ellas solo del $10-15 \%$ se localizan en el sistema nervioso. Por otro lado, los tuberculomas son las lesiones menos frecuentes y constituyen solo el $1 \%$ de la TB de forma global.

Estos granulomas se producen por reactivación a partir de TB primaria latente, en la generación de este proceso se señalan varios factores de riesgo, dentro de ellos tenemos: nueva exposición al Mycobacterium tuberculosis, enfermedades pulmonares crónicas, insuficiencia renal crónica, diabetes mellitus, tabaco, predisposición genética, cáncer, enfermedades hematológicas como linfoma o leucemia, inmunosupresión, uso de drogas intravenosas, residentes o trabajadores de lugares con congregación de personas (penitenciarias, trabajadores de servicios de salud, laboratorios microbiológicos, etcétera), entre otros ${ }^{6}$.

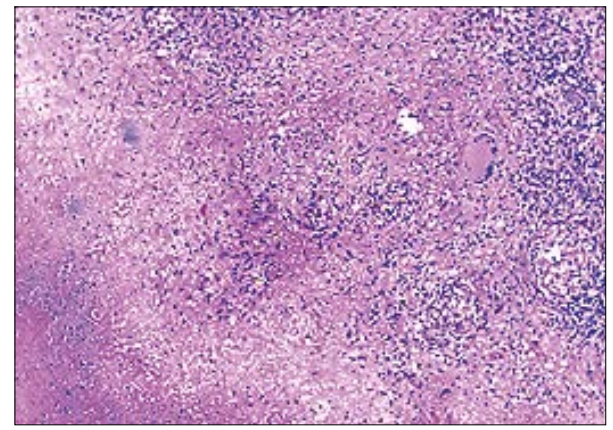

Figura 3. Técnica de coloración de hematoxilina y eosina x 100. Inflamación granulomatosa crónica con presencia de células gigantes y granulomas epitelioides con necrosis caseosa central.

Los tuberculomas cerebrales habitualmente muestran manifestaciones clínicas inespecíficas. Cuando se presentan en pacientes sin signos de lesiones pulmonares o de otros aparatos o sistemas sugerentes de TB constituyen un reto diagnóstico ${ }^{4}$. El diagnóstico diferencial incluye fundamentalmente a las neoplasias y a otros granulomas del encéfalo, los cuales pueden tener múltiples causas, a saber: infecciosas (bacterianos o micóticos), parasitarios e inflamatorias (Tabla 1).

Según Salaskar et $\mathrm{al}^{12}$ y Monteiro et $\mathrm{al}^{8}$ hay varios aspectos que dificultan el diagnóstico inicial de tuberculoma, los cuales confluyeron en el caso presentado: inmunocompetencia del paciente, así como ausencia de signos sugerentes de tuberculosis pulmonar o diseminada.

A pesar de no contar con signos patognomónicos, los estudios neuroimagenológicos, sobre todo la RM, son indispensables en el proceso diagnóstico de estos enfermos. Habitualmente se presentan con las llamadas "lesiones en anillo", más evidentes en la secuencia de $\mathrm{T}_{1}$ contrastada, las mismas pueden ser detectadas en otros tipos de lesiones: neoplásicas, infecciosas, vasculares, desmielinizantes, entre otras, según se resumen en la Tabla $2^{15}$.

Algunos signos en la RM se han relacionado en mayor medida con algunas variedades de granulomas intracraneales. En casos de tuberculoma es más frecuente la detección de lesiones solitarias, solo el $15-33 \%$ de los casos muestran lesiones múltiples, habitualmente relacionadas con Síndrome de Inmunodeficiencia Adquirido (SIDA). Es también característico cuando se observa el "signo de la diana", aunque solo se detecta en un tercio de los pacientes, el mismo se observa en la TC cerebral como una calcificación central dentro de una lesión que capta contraste en anillo. En el caso de la RM se observa un centro hipointenso y periferia hiperintensa en $T_{2}$. En la descripción de dicho signo existe una variedad "excéntrica", evidente en $T_{1}$ contrastada como una pequeña zona nodular, hiperintensa y excéntrica, al interior de una lesión en anillo, y otra "concéntrica", evidente en $T_{2}$ o FLAIR como una zona central hipointensa, con hiperintensidad en la periferia y otra zona hipointensa perimetral a toda la lesión ${ }^{7}$.

Morales et $\mathrm{al}^{9} \mathrm{y}$ Jhaveri et $\mathrm{al}^{3}$ fundamentan que la detección de picos elevados de lípidos en el examen con espectroscopía cerebral por RM, aunque pueden detectarse en gliomas, linfomas o abscesos (en estos últimos también se registra elevación de aminoácidos), sugiere el diagnóstico de 


\begin{tabular}{|c|c|}
\hline \multicolumn{2}{|r|}{ Tabla 1. Principales causas de granulomas intracraneales } \\
\hline Causas & Especificaciones \\
\hline Infecciosas & $\begin{array}{l}\text { Bacterianas: Mycobacterium tuberculosis*, Treponema pallidum } \\
\text { Micóticas: Aspergillus spp, Candida spp, Histoplasma capsulatum, Blastomyces spp, Cryptococcus neo- } \\
\text { formans/gatti, Paracoccidioides spp. }\end{array}$ \\
\hline Parasitarias & Taenia solium (neurocisticercosis), Toxoplasma gondi, Trypanosoma cruzi (Enfermedad de Chagas) \\
\hline Protozoos & Amebas de vida libre (Acanthamoeba spp, Balamathia madrillaris, Sappinia pedata) \\
\hline Inflamatorias & $\begin{array}{l}\text { Cuerpo extraño intracraneal }{ }^{*} \text {, Neurosarcoidosis, Enfermedad de Wegener*, Enfermedad granulomatosa } \\
\text { crónica, Granulomatosis con poliangeítis }\end{array}$ \\
\hline
\end{tabular}

\begin{tabular}{|c|c|}
\hline Causas & Tipos anatomo clínicos \\
\hline Neoplasias & $\begin{array}{l}\text { Primarias: glioma de alto grado de malignidad, Linfoma* } \\
\text { Metástasis }\end{array}$ \\
\hline Infecciosas & $\begin{array}{l}\text { Absceso cerebral piógeno, leucoencefalopatía multifocal pro- } \\
\text { gresiva* }^{*}\end{array}$ \\
\hline $\begin{array}{l}\text { Granulomas } \\
\text { (ver tabla 1) }\end{array}$ & 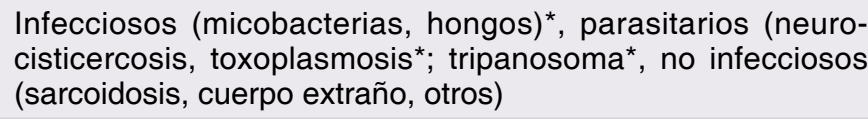 \\
\hline Otros tumores quísticos & $\begin{array}{l}\text { Quistes: aracnoideo, dermoides, epidermoides, neuroentérico, } \\
\text { neuroepitelial. Craneofaringioma adamantimomatoso, variantes } \\
\text { poco frecuentes de meningiomas y neurofibromas }\end{array}$ \\
\hline Vasculares & Hematomas en reabsorción \\
\hline Post radioterapia & Radionecrosis \\
\hline Desmielinizante & Esclerosis concéntrica de Balo \\
\hline
\end{tabular}

tuberculoma.

Como resultado de que los pacientes que se presentan de forma exclusiva con tuberculomas cerebrales, como en el caso presentado, son paucibacilares, el diagnóstico positivo es habitualmente histopatológico, donde se revela un granuloma con necrosis caseosa. Debido a que los tuberculomas pueden coincidir con meningitis tuberculosa puede detectarse en el LCR pleocitosis a predominio linfocítico y niveles de la ADA mayores a 5,8 U/I, hecho que también puede ser registrado en linfomas y meningitis micóticas. En contraparte hay que tener en cuenta los probables riesgos de realizar una punción lumbar para el examen del LCR en pacientes con lesiones intracraneales con efecto de masa.

La observación directa con tinción para bacilos ácido alcohol resistente (BAAR) en el tejido o LCR es positiva en menos del $10 \%$ de los casos, aún más difícil si se ha fijado el tejido con formaldehido. El cultivo continúa siendo el "gold estándar" del diagnóstico, el mismo ayuda a diferenciar estas lesiones de las infecciones por otras micobacterias y a realizar estudio de sensibilidad antimicrobiana; sin embargo, para que sea positivo se requieren concentraciones en el tejido analizado de al menos 100 bacterias por $\mathrm{ml}$, además demora entre 2-6 semanas ${ }^{5}$.

En la actualidad resulta de gran ayuda el complemento diagnóstico con técnicas de identificación bacteriana median- te amplificación del material genético (PCR), las cuales pueden identificar el micobacterium tuberculoso con la presencia de solo 6 copias por $\mathrm{ml}$ en el tejido analizado, el resultado está disponible en solo 2 horas y es posible determinar la existencia de resistencia al tratamiento con Rifampicina ${ }^{1,2}$.

De particular relevancia en la paciente presentada es el hecho de cursar con embarazo del segundo trimestre, casos similares son escasos en la literatura médica. Nomani et al ${ }^{10}$ describieron un caso en el 2017 y encontraron el reporte de solo 7 pacientes previas. Aunque no totalmente aceptado, algunos autores plantean que la depresión relativa de la inmunidad celular durante el embarazo puede contribuir a la generación de estas lesiones. Por otro lado, no existe duda de que cualquier forma de TB en el embarazo aumenta la mortalidad materna, así como el índice de prematuridad, bajo peso al nacer y la mortalidad neonatal. Durante este período el tratamiento adquiere también particularidades, la realización de craneotomía y resección de la lesión no está contraindicada, pero requiere de una planificación pormenorizada desde el punto de vista anestésico, quirúrgico y obstétrico que contempla evitar fármacos ototóxicos, como la estreptomicina, en el tratamiento antituberculoso. Afortunadamente la transmisión vertical de la madre enferma al feto es rara.

La historia natural de los tuberculomas cerebrales comprende una mortalidad que supera el $80 \%$ de los casos si no 
se implementa el tratamiento de forma adecuada. Los fármacos antituberculosos constituyen la primera línea terapéutica y pueden complementarse con antiepilépticos y esteroides según la aparición de crisis comiciales y al grado de edema perilesional respectivamente. La resección quirúrgica de la lesión se indica cuando existe importante efecto de masa, mala respuesta al tratamiento farmacológico o incertidumbre diagnóstica ${ }^{13}$.

Se ha comprobado la importancia de la agilidad en el diagnóstico y el tratamiento para obtener buenos resultados terapéuticos en estos enfermos. En el caso presentado la decisión de la intervención sin mayor dilación, a pesar de la coexistencia de embarazo, fue relevante para el tratamiento oportuno y apoya el hecho, bien documentado en la literatura, de la posibilidad de realización exitosa de intervenciones neuroquirúrgicas en el embarazo cuando se planifican adecuadamente por un equipo multidisciplinario.

\section{Conclusiones}

Los tuberculomas cerebrales como manifestación exclusiva de tuberculosis en el embarazo son lesiones raras pero probables. La sospecha clínica, la confirmación diagnóstica y la cirugía en los casos indicados, llevados a cabo con agilidad y planificados por un equipo multidisciplinario, son relevantes para la obtención de buenos resultados terapéuticos, tanto para la madre como para el feto.

\section{Referencias}

1. Babafemi OE, Cherian PB, Banting L, Mills AG, Ngianga K. Effectiveness of real-time polymerase chain reaction assay for the detection of Mycobacterium Tuberculosis in pathological simples: a systematic review and meta-analysis. Systematic Reviews. 2017; 16: 215-36. DOI: 10.1186/s13643-017-0608-2.

2. Bankar S, Set R, Sharma D, Shah D, Shastri J. Diagnostic accuracy of Xpert MTB/RIF assay in extrapulmonary tuberculosis Indian J Med Microbiol [internet] 2018 [citado 13 de agosto 2019]; 36: 357-63. Disponible en: http: //www.jjmm.org/text. asp?2018/36/3/357/245383.

3. Jhaveri DM, Harder LS. Tuberculosis. En: Osborn GA, Digne BK, eds. Imagen en Neurología. Elsevier. 2018.p. 180.
4. Kim JK, Jung TY, Lee KH, Kim KS. Radiological Follow-up of a Cerebral Tuberculoma with a Paradoxical Response Mimicking a Brain Tumor. J Korean Neurosurg Soc. 2015; 57 (4): 307-10. Doi: 10.3340/jkns.2015.57.4307.

5. Lewinsohn MD, Leonard KM, LoBre AP, Cohn LD, Doley LCh, Desmond E, et al. ATS/CDC/IDSA. Clinical Practice Guidelines: Diagnosis of Tberculosis in Adults and Children. Clin Infect Dis. 2017; 64 (2): 111-15. DOI: 10.1093/cid/cid/ciw778.

6. Loddenkemper R, Lipman M, Zumla A. Clinical Aspects of Adult Tuberculosis. Cold Spring Harb Perspect Med. 2016; 6: a017848. DOI: 10.1101/cshperspect.a017848.

7. Mahadevan A, Hanumantapura RA, Parthasarathy S, Nath A, Ranga U, Sucarla K. Neuropathological correlate of the "concentric target singn" in MRI of HIV associated cerebral toxoplasmosis. J magn Reson Imaging. 2013; 38 (2): 488-95. Doi: 10.1002/ jmri.24036.

8. Monteriro R, Carneiro CJ, Costa C, Duarte R. Cerebral tuberculoma-A clinical Challenge. Respiratory Medicine Case Report. 2013; 9: 34-37. Doi: 10.1016/j_rmcr.2013.04.003.

9. Morales H, Alfaro D, Mertinot C, Fayed N, Gaskill-Shipley M. MR spectroscopy if intracranial tuberculoma: A singlet peak at $3.8 \mathrm{ppm}$ as potential marker to differentiate them from malignant tumors. Neuroradiol J. 2015; 28 (3): 294-302. Doi: 10.1177/1971400915592077.

10. Namani S, Dreshaj S, Zogaj A. Tuberculous meningoencephalitis associated with brain tuberculomas during pregnancy: a case report. Journal of Medical Case Reports. 2017; 11: 175. Doi: 10.1186/s13256-017-1347-7.

11. Ramírez-Lapausa M, Menéndez-Saldaña A, Noquerado-Asensio A. Tuberculosis extrapulmonar, una revisión. Rev Esp Sanid Penit. 2015; 17: 3-11.

12. Salaskar AL, Hassaneen W, Keenan $\mathrm{CH}$, Suki D. Intracranial tuberculoma mimicking brain metástasis. J Can Res Ther. 2015; 11: 653-56. Doi: 10.4103/09731482.138131.

13. Saleh M, Saeedi AA, Pooran AA. Brain Tuberculomas: A Case Report. Jundishapur J Microbiol. 2014; 7 (7): e11252. Doi: 10.5812/jjm.11252.

14. Shivakoti R, Sharma D, Mamon G, Pham K. Association of HIV infection with extrapulmonary tuberculosis: a systematic review. Infection. 2017; 45 (1): 11-21. Doi: 10.1007/s15010-016-0960-5.

15. Yuzuwa H, Hirose $Y$, Kimura $T$, Kimura S, Sugwara $H$, Yanagisawa $A$, et al. A case of cerebral tuberculoma mimicking neurocysticercosis. Acute Medicine and Surgery. 2017; 4: 329-33. Doi: 10.1002/cms2.272. 